\title{
Atrial myxoma: tumour or trauma?
}

James Nolan, Pauline J Carder, Peter Bloomfield

\begin{abstract}
A mass lesion developed in the right atrium at the site of a trans-septal puncture after percutaneous balloon dilatation of the mitral valve in a man aged 74. The lesion had the pathological appearance of an atrial myxoma and seemed to have developed after trauma to the intra-atrial septum.

This case suggests that at least some atrial myxomas are reactive rather than neoplastic in origin.
\end{abstract}

\section{Case report}

The patient, a 74 year old man, had acute rheumatic fever as a child. He presented with heart failure and atrial fibrillation aged 61 . Mitral stenosis was diagnosed and he was treated successfully with digoxin, diuretics, and warfarin.

Signs and symptoms of mitral stenosis progressed over the next 11 years until he was admitted to hospital in April 1988 aged 72 with breathlessness at rest and signs of congestive cardiac failure. Cross sectional echocardiography confirmed severe calcified mitral stenosis with an enlarged left atrium, right ventricle, and right atrium. No other intracardiac abnormalities were detected (fig 1A). His poor general condition and severe chronic obstructive pulmonary disease (forced expiratory volume in the first second 0.7 litres) was thought to preclude surgical valve replacement and he was considered for balloon dilatation of the mitral valve. As part of his assessment for valve dilatation multiple echocardiographic views of both atria and the intra-atrial septum were obtained to exclude the presence of intra-atrial clot. No abnormality was detected within either atrium at this time.

Percutaneous balloon dilatation of the mitral valve was performed on 7 April 1988 . The haemodynamic findings were mean transmitral gradient $11 \mathrm{~mm} \mathrm{Hg}$, pulmonary artery
Department of Cardiology, Royal Infirmary, Edinburgh J Nolan

P Bloomfield

Department of

Pathology, University of Edinburgh

P Carder

Correspondence to Dr J Nolan, Department of Cardiology, Royal Infirmary, Edinburgh EH3 9YW
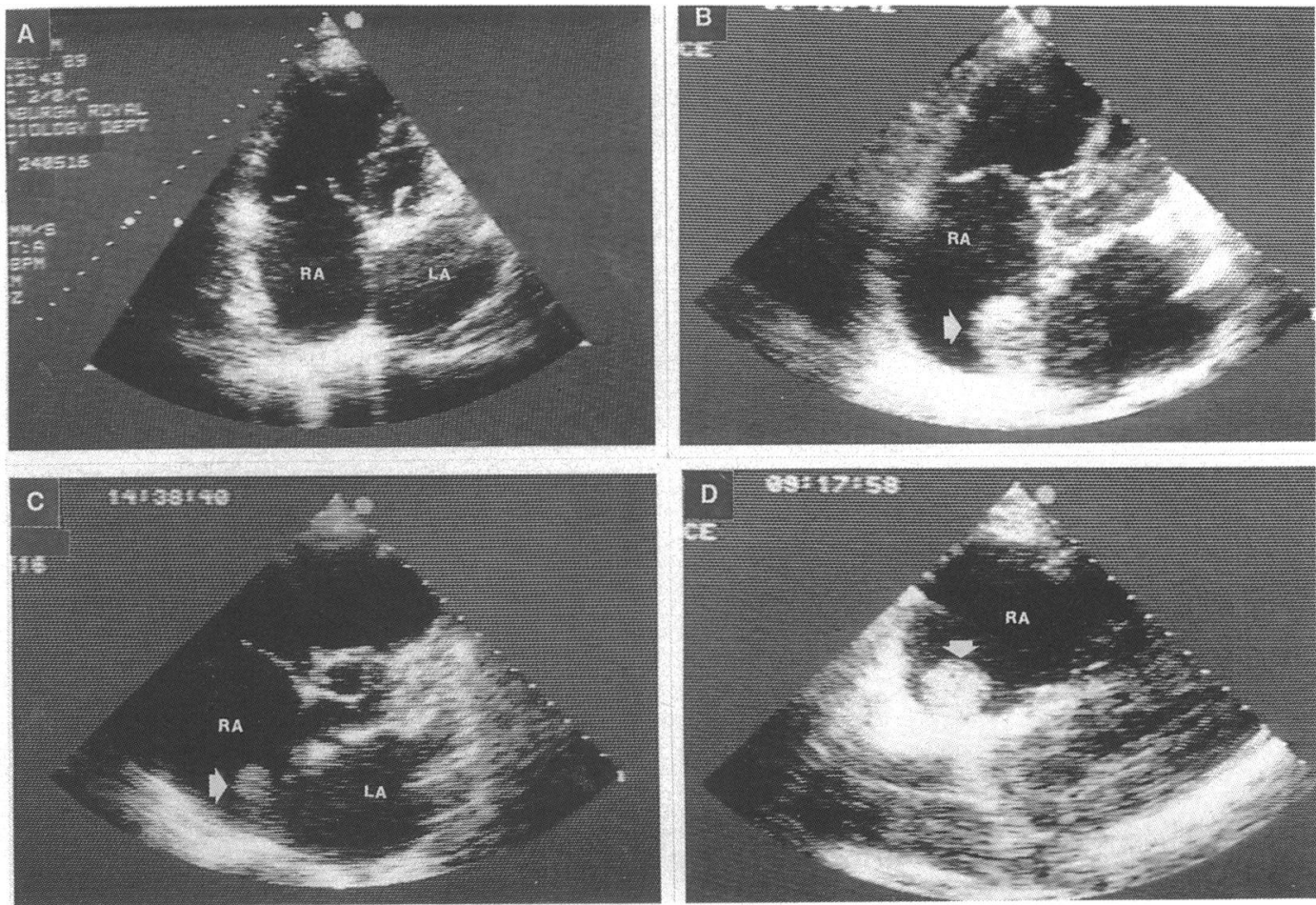

Figure 1 (A) Apical four chamber echocardiogram obtained before balloon dilatation of the mitral valve showing a heavily calcified rheumatic mitral valve, a large left atrium $(L A)$, volume loaded right ventricle and right atrium ( $R A$ ), but no evidence of a right atrial mass lesion. (B) Preoperative apical four chamber echocardiogram showing a large mass lesion in the right atrium (RA) attached to the intra-atrial septum (white arrow). (C) Preoperative parasternal short axis echocardiogram showing a mass lesion (white arrow) in the right atrium $(R A)$. (D) Preoperative subcostal echocardiogram showing a mass lesion (white arrow) in right atrium $(R A)$. 

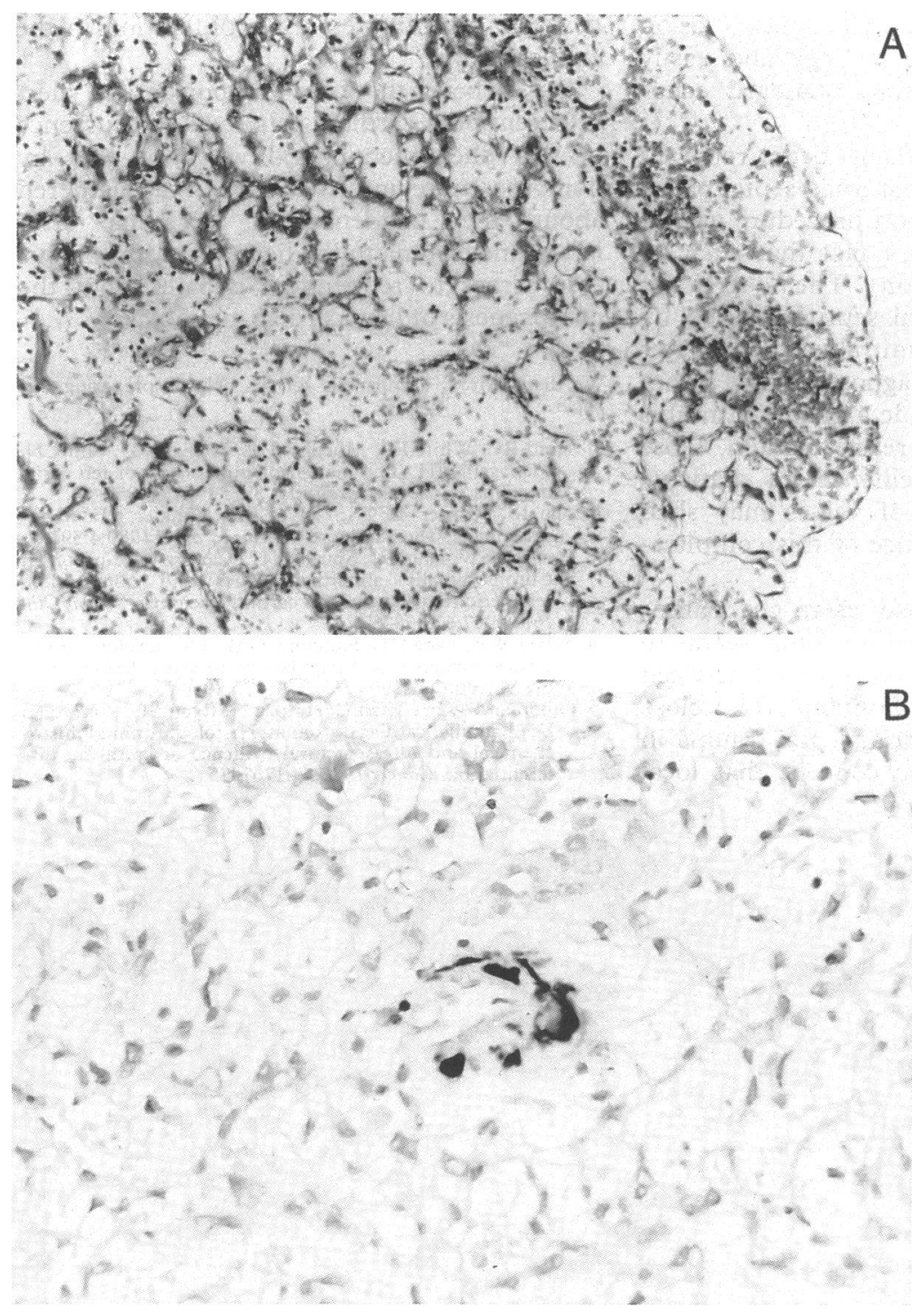

Figure 2 (A) Complex trabecular network of vascular strands within the atrial myxoma (original magnification $\times 40$, haematoxylin and eosin stain). (B) Positive immunohistochemical staining for CAM 5.2, a marker of epithelial differentiation (original magnification, $\times 100, C A M 5 \cdot 2$ ).

pressure $66 / 30 \mathrm{~mm} \mathrm{Hg}$, and cardiac index $2.231 / \mathrm{min} / \mathrm{m}^{2}$. The coronary arteries were normal and left ventricular function was good. The procedure was initially uneventful, but after three dilatations of the mitral valve acute tamponade developed owing to a perforation of the left ventricle. Emergency thoracotomy was undertaken and a left ventricular tear was repaired successfully. The patient made a satisfactory recovery from this procedure and was subsequently discharged home. At follow up in the outpatient clinic his exercise capacity remained unacceptably limited by his unrelieved severe mitral stenosis.

Because of his persisting severe symptoms the patient was considered for elective mitral valve replacement. As part of his preoperative assessment echocardiography was repeated on 27 April 1989. This study showed a pedunculated lesion in the right atrium attached to the intra-atrial septum at the site of the previous trans-septal puncture. This lesion was large, easily visible, and present in multiple views (fig $1 \mathrm{~B}, \mathrm{C}$, and $\mathrm{D}$ ).
At operation on 7 August 1989 his heavily calcified stenotic mitral valve was replaced with a Carpentier-Edwards prosthesis. Within the right atrium was a pedunculated mass measuring $3 \mathrm{~cm}$ by $2 \mathrm{~cm}$ attached to the intraatrial septum at an area of obviously abnormal endothelium thought to be the site of the trans-septal catheterisation. The mass and the abnormal endothelium were resected uneventfully.

\section{PATHOLOGY}

Histological examination of the resected tissue showed a somewhat lobulated contour to a lesion containing polygonal and stellate cells, forming cords, clusters, and vascular channels within a loose myxoid matrix. In some areas there were dense trabecular networks of intercommunicating strands (fig 2A). There was focal haematopoiesis and the stroma was alcianophilic. Immunohistochemistry showed positive staining for Factor VIIIrAg (Dako, Buckinghamshire) which was most prominent in the lining of the vascular channels. There was widespread positivity for vimentin (Dako) and intense staining of single cells for PGP 9.5 (Ultraclone, Cambridge), and NSE (Dako). There was focal positivity for CAM $5 \cdot 2$ (Becton Dickinson, Oxford), a marker of epithelial differentiation (fig 2B). The appearances were those of an atrial myxoma.

\section{Discussion}

There is still controversy about the pathogenesis of atrial myxoma. Occasional cases with chest wall invasion, ${ }^{1}$ distant metastases, or chromosomal abnormalities, ${ }^{3}$ suggest that some at least are neoplastic. Histologically, however, atrial myxoma has features in common with organising thrombus and some workers maintain that it is essentially a reactive proliferative phenomenon. ${ }^{4}$ Indeed distinguishing myxoma from thrombus can occasionally pose problems for the pathologist. Myxomas have also been reported to show osseous, chondroid, and even glandular ${ }^{5}$ differentiation.

The myxoma in our patient, as well as having a population of bizarre mesenchymal cells within a loose myxoid matrix, also contained foci of haematopoiesis and had immunohistochemical evidence of epithelial differentiation. Such features would be most unlikely in thrombus and warrant a diagnosis of myxoma.

This right atrial myxoma seems to have developed after iatrogenic trauma to the atrial septum. The lesion seems to have developed rapidly over a 12 month period between the pre-dilatation and preoperative echocardiograms. It is unlikely that the lesion was present but not seen on the echocardiogram before the attempted balloon dilatation. Neither of the two separate echocardiographic examinations performed before the procedure with multiple views of both atria and the intra-atrial septum showed a lesion. All the echocardiographic recordings in this patient were performed by the same experienced 
operator. Furthermore, the mass lesion subsequently detected was large and easily visualised in three separate standard echocardiographic views.

This association has not been reported before, despite the fact that trans-septal puncture is a relatively common procedure worldwide; this may reflect the fact that such an association is uncommon. The degree of trauma to the intra-atrial septum caused by balloon dilatation of the mitral valve is greater than that occurring in diagnostic septal punctures and this may provide a greater stimulus for the development of a reactive lesion. Close echocardiographic surveillance of patients after balloon dilatation of valves may shed more light on the incidence of this complication.

This lesion, which had clear cut pathological features of an atrial myxoma, seems to have developed in a 12 month period between two consecutive echocardiograms. The close temporal relation with atrial septal trauma in this patient supports the concept that local tissue injury initiates cell proliferation in the endocardium.

We believe that it is important to alert interventional cardiologists to the occurrence of this phenomenon and we hope that by doing so more information will be gathered about the incidence of myxoma after iatrogenic trauma to the heart. In addition our case serves to reopen the debate about the pathogenesis of intracardiac myxomas.

We thank Mr C Campanella for his help in preparing thi paper.

1 Read RC, White HJ, Murphy ML, Williams D, Sun CN Flanagan WH. The malignant potentiality of left atria myxoma. J Thorac Cardiovasc Surg 1974;68:857-68.

2 Seo IS, Warner TFCS, Colyer RA, Winkler RF. Metastasizing atrial myxoma. Am J Surg Pathol 1980;4:391-9. 3 Dewald GW, Dahl RJ, Spirbeck JL, Carney JA, Gordon H. Chromosomally abnormal clones and non-random telomeric translocations in cardiac myxomas. Mayo Clin Proc 1987;62:558-67.

Salyer WR, Page DL, Hutchins GM. The development of cardiac myxomas and papillary endocardial lesions from mural thrombus. Am Heart J 1975;89:4-17

5 Goldman BI, Frydman C, Harpaz N, Ryan SF, Loiterman D. Glandular cardiac myxomas. Histologic immunohistochemical and ultrastructural evidence of epithelial differentiation. Cancer 1987;59:1767-75. 